COMPUTER METHODS IN APPLIED MECHANICS AND ENGINEERING 36 (1983) 257-276

NORTH-HOLLAND PUBLISHING COMPANY

\title{
SEMI-RADIAL SINGULARITY MAPPING THEORY FOR LINE SINGULARITIES IN FRACTURE MECHANICS
}

\author{
Masayuki OKABE \\ Mitsui Mining and Smelting Co., Ltd., Chuo-Ku, Tokyo, Japan \\ Noboru KIKUCHI \\ Department of Mechanical Engineering and Applied Mechanics, University of Michigan, \\ Ann Arbor, MI 48109, U.S.A. \\ Received 2 March 1981 \\ Revised manuscript received 15 June 1982
}

\begin{abstract}
Fundamental theory of the semi-radial singularity mapping dealing with line singularities in the stress and strain is presented. Explicitly the brick-type semi-radial singularity mapping element as well as the wedge-type one are proposed. Element familization is then described based on the conventional polynomial interpolations for the cube and simplex, with examinations of the elaborate trial function spaces.
\end{abstract}

\section{Introduction}

A variety of finite element interpolations have been proposed not only for the cube [1-6] but also for the simplex [7-12]. In the singularity elementology with required stress and strain singularities those polynomial interpolations are used only to prescribe the element geometry, or reversely only to define the approximated displacement field.

In two-dimensional fracture mechanics, for example, we have plenty of crack elements with some peculiar trial functions and polynomial parametric representations [13-16]. A series of finite elements with the polynomial trial functions under the singularity mapping are also presented $[12,17-21]$. We note in the latter that any higher order elements can easily and systematically be produced by the known polynomial interpolations.

In three-dimensional fracture mechanics treatment of the line (not the point) singularities are significant. For such through-wall crack, we have the superposed finite elements by Yamamoto and Sumi [22] and Yagawa and Nishioka [23], utilizing the global-local approximations of analytical asymptotic and normal isoparametric modes. In more orthodox finite element form Stern [16], Akin [24] and Hughes and Akin [25] presented the peculiar singular trial functions over wedge-type elements with the collapse of a plane of the normalized 3-cube. As an alternative mapping applied to line singularities we have only the quarter-point mapping by Ingraffea and Manu [26] also with collapse.

In this paper we concentrate on the fundamentals of the semi-radial singularity mapping for line singularities. Namely the general theory established in two dimensions associated with point singularities $[12,21]$ is modified so as to deal unifiedly with the brick-type and 
wedge-type elements. We explicitly present the semi-radial singularity mapping for originally 8 -node brick and 6-node wedge elements with the proof of the realized singularities and strain energy finiteness.

Then we familize our singularity elements by applying the conventional polynomial interpolations with examinations of the created trial function spaces. Especially for wedge elements we utilize not only the collapsed rectangular interpolations widely used in fracture mechanics but also the well-known triangular interpolations.

\section{Trial and mapping function spaces associated with line singularities}

We consider a general finite element composed of $n$ nodes depicted in Fig. 1 in the global Cartesian system $(x, y, z)$, which is normalized into the $(-1,1)$ cuboid in the local parametric system $(\xi, \eta, \zeta)$. In the general form of Lagrange interpolation the trial function kept over the element can be expressed as

$$
\phi=\sum_{i=1}^{n} \phi_{i} N_{i}(\xi, \eta, \zeta)
$$

where $\phi_{i}$ denotes the $\phi$-value at node $i$. The field shape functions $N_{i}$ should satisfy

$$
N_{i}\left(\xi_{j}, \eta_{j}, \zeta_{j}\right)=\delta_{i j}
$$

Here $\delta_{i j}$ is the Kronecker delta, and $\xi_{j}, \eta_{j}$ and $\zeta_{i}$ denote the parametric coordinates of node $j$. The trial function space $F^{2}$ then uniquely defined as

$$
\mathscr{F}=\left\{f: f=\sum_{i=1}^{n} f_{i} N_{i}\right\}
$$

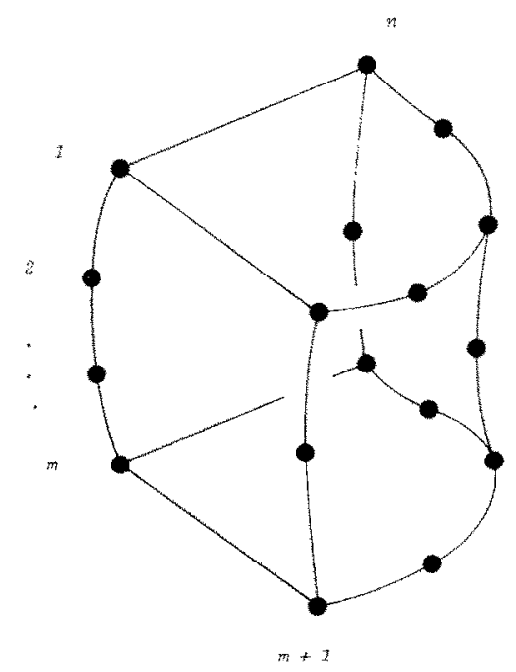

Fig. 1. Original $n$ wnode element geometry in the global Cartesian system $(x, y, z)$. Line singularities exist along the central edge composed of nodes 1 to $\mathrm{m}$. Other side and face nodes are allowed only on the opposite surface. 
where $f$ and $f_{i}$ represent an arbitrary, sufficiently smooth function and its nodal value at $i$, respectively.

In the practical finite element analysis the trial function space $\mathscr{F}$ originally defined in $(\xi, \eta, \zeta)$ should be recharacterized in $(x, y, z)$ through the parametric representation of the form

$$
x=\sum_{i=1}^{n} x_{i} M_{i}(\xi, \eta, \zeta)
$$

where

$$
\boldsymbol{x}=[x, y, z] \text { and } \boldsymbol{x}_{i}=\left[x_{i}, y_{i}, z_{i}\right] .
$$

Here $\boldsymbol{x}_{i}$ denotes the Cartesian coordinates of node $i$. The mapping shape functions $M_{i}$ satisfy

$$
M_{i}\left(\xi_{j}, \eta_{j}, \zeta_{j}\right)=\delta_{i j}
$$

and hence the mapping function space $M$ can uniquely be defined as

$$
\mathcal{M}=\left\{f: f=\sum_{i=1}^{n} f_{i} M_{i}\right\} .
$$

In the isoparametric mapping we preserve the identity of the trial and mapping function spaces by

$$
N_{i}=M_{i}, \quad i=1, \ldots, n,
$$

in order to realize the $x$-reproducibility of the form

$$
x=\sum_{i=1}^{n} x_{i} N_{i} .
$$

In linear fracture mechanics, on the other hand, the trial function space is to be different from the mapping function space, since some appropriate singularities are needed in the first derivatives of our trial function, say in the stress and strain. The conventional polynomial interpolations are thus applicable only to the field shape functions, or only to the mapping shape functions. We restrict ourselves in this paper to the use of the polynomial trial function space in $(\xi, \eta, \zeta)$. Namely, our objective is to develop the peculiar singularity mapping function space. Strictly the trial function space should be made for all the displacement components, say $u, v$ and $w$. However, for simplicity, we use only the scalar trial function $\phi$ throughout this paper.

The singularities exist along the central edge of the original element of Fig. 1, which is composed of nodes $1, \ldots, m$. Any curved central edge is allowed for generality. We normalize the $n$-node element into the $(-1,1)$ cube of Fig. 2 parametrically in $(\xi, \eta, \zeta)$. An alternative normalization illustrated in Fig. 3 with collapse of the $\xi=-1$ surface into the central edge is also dealt with. In the former, any side nodes are allowed on the central edge of $\xi=\eta=-1$ with side and face nodes on the opposite surfaces of $\xi=1$ and $\eta=1$. In the latter, any side and face nodes can be placed on the opposite surface of $\xi=1$ with side nodes on the central edge. 


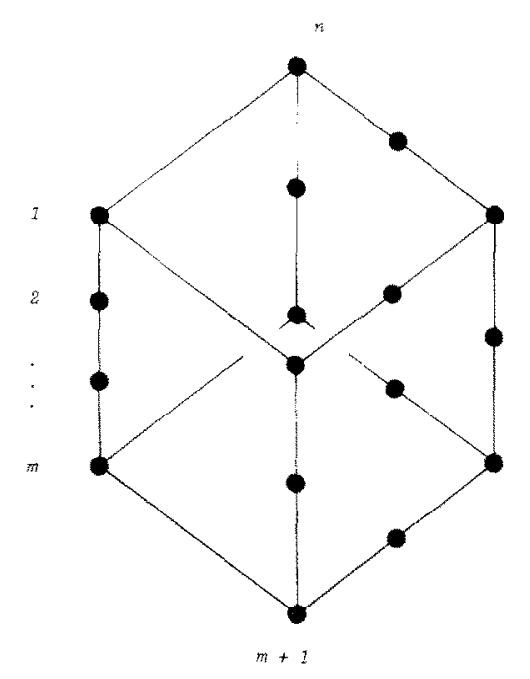

Fig. 2. Normalized 3-cube in the local parametric system $(\xi, \eta, \zeta)$. On the opposite surfaces of $\xi=1$ and $\eta=1$ any side and face nodes can be placed. The central edge of $\xi=\eta=-1$ is composed of $m$ nodes.

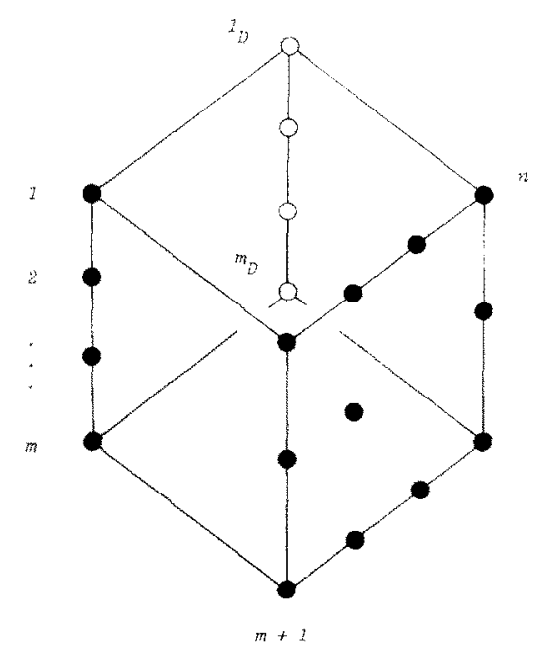

Fig. 3. Normalized cube in the local parametric system $(\xi, \eta, \zeta)$ with collapse of the $\xi=-1$ plane into the central edge of $\xi=\eta=-1$. On the opposite surface of $\xi=1$ any side and face nodes are allowed. The central edge after collapse is composed of $m$ nodes.

Naturally on the central edge of $\xi=\eta=-1$

$$
x_{0}(\zeta)=\sum_{i=1}^{m} x_{i} M_{i}(-1,-1, \zeta)
$$

where $\boldsymbol{x}_{0}$ denotes the Cartesian coordinates of a point on the central edge by

$$
\boldsymbol{x}_{0}=\left[x_{0}, y_{0}, z_{0}\right]
$$

Let $r$ denote a vector from $\left(x_{0}, y_{0}\right)$ to $(x, y)$ such that

$$
r=\left[x-x_{0}, y-y_{0}\right]
$$

Then the two-dimensional radius $r$ emanating from $\left(x_{0}, y_{0}\right)$ can be written as

$$
r=\|\boldsymbol{r}\|=\left\{\left(x-x_{0}\right)^{2}+\left(y-y_{0}\right)^{2}\right\}^{1 / 2}
$$

In our problem $O\left(r^{\lambda-1}\right)$ line singularities are needed in the first derivatives $\partial \phi / \partial x$ and $\partial \phi / \partial y$, say in the stress and strain along the central edge. Here $\lambda$ is the singularity constant, which is positive below unity $(0<\lambda<1)$.

\section{Relation between Euclidean and nondimensional radii}

Using (4) the Fuclidean radius $r$ of (13) can be expressed as 


$$
\begin{aligned}
r= & \left\{\left[\sum_{i=1}^{m} x_{i}\left\{M_{i}(\xi, \eta, \zeta)-M_{i}(-1,-1, \zeta)\right\}\right]^{2}+\left[\sum_{i>m} x_{i} M_{i}(\xi, \eta, \zeta)\right]^{2}\right. \\
& \left.+\left[\sum_{i=1}^{m} y_{i}\left\{M_{i}(\xi, \eta, \zeta)-M_{i}(-1,-1, \zeta)\right\}\right]^{2}+\left[\sum_{i>m} y_{i} M_{i}(\xi, \eta, \zeta)\right]^{2}\right\}^{1 / 2}
\end{aligned}
$$

Naturally (10) can be generalized as

$$
x_{0}(\zeta) \sum_{i=1}^{m} M_{i}(\xi, \eta, \zeta)=\sum_{i=1}^{m} x_{i} M_{i}(\xi, \eta, \zeta)
$$

and hence (14) can further be written as

$$
r=\left[\left\{\sum_{i>m}\left(x_{i}-x_{0}\right) M_{i}\right\}^{2}+\left\{\sum_{i>m}\left(y_{i}-y_{0}\right) M_{i}\right\}^{2}\right]^{1 / 2}
$$

On behalf of the Euclidean radius $r$, we introduce the nondimensional radius $\rho$ so that it takes zero on the central edge and unity on the opposite surface. Let us embed the constant and $\rho$ terms in our mapping function space such that

$$
\begin{aligned}
& 1=\sum_{i=1}^{n} M_{i}, \\
& \rho=\sum_{i>m} M_{i} .
\end{aligned}
$$

Then the nondimensional radius can be written as

$$
\rho=1-\sum_{i=1}^{m} M_{i}
$$

We introduce here the $M_{i}^{*}$ functions by

$$
M_{i}^{*}=\rho \cdot{ }^{1} M_{i}, \quad i>m .
$$

Substitution of (20) into (16) then gives the relation between $r$ and $\rho$ of the form

$$
r=\rho\left[\left\{\sum_{i>m}\left(x_{i}-x_{0}\right) M_{i}^{*}\right\}^{2}+\left\{\sum_{i>m}\left(y_{i}-y_{0}\right) M_{i}^{*}\right\}^{2}\right]^{1 / 2}
$$

If all the $M_{i}^{*}$ functions by (20) are bounded throughout the region of interest especially on the central edge, then $\rho$ is proportional to $r$ at least within the vicinity of the central edge, i.e. $O(r)=O(\rho)$. The singularities may thus sufficiently be treated by $\rho$ instead of $r$.

Obviously $M_{i}^{*}$ should satisfy

$$
M_{i}^{*}\left(\xi_{j}, \eta_{j}, \zeta_{j}\right)=\delta_{i j}, \quad i>m
$$


and

$$
1=\sum_{i>m} M_{i}^{*}
$$

We further substitute (10) into (15). Then we have

$$
M_{i}(\xi, \eta, \zeta)=M_{i}(-1,-1, \zeta) \sum_{i=1}^{m} M_{j}(\xi, \eta, \zeta), \quad i=1, \ldots, m
$$

Using (19), (24) can be rewritten as

$$
M_{i}(\xi, \eta, \zeta)=(1-\rho) M_{i}(-1,-1, \zeta), \quad i=1, \ldots, m .
$$

\section{The semi-radial singularity mapping for line singularities}

Expecting $O\left(\rho^{\lambda-1}\right)$ derivative singularities we impose the semi-radial field condition on the trial function of (1) by

$$
\rho^{\lambda}=\sum_{i>m} N_{i},
$$

which guarantees that the $\rho^{\lambda}$ term is reproducible. Since $\lambda$ is positive below unity the displacement field has no singularity. We also realize the constant field condition as usual such that

$$
1=\sum_{i=1}^{n} N_{i}
$$

Then the nondimensional radius $\rho$ can be defined as

$$
\rho^{\lambda}=1-\sum_{i=1}^{m} N_{i}
$$

In order to assure that the lowest terms associated with the nondimensional radius are $\rho^{\wedge}$ (except for $\rho^{0}$ ) we must restrict the other field shape functions also. Let us introduce

$$
N_{i}^{*}=\rho^{-\lambda} N_{i}, \quad i>m .
$$

Then noting that each $N_{i}(i>m)$ takes the value zero on the central edge, $N_{i}^{*}$ should be bounded everywhere. Furthermore, the following hold

$$
\begin{aligned}
& N_{i}^{*}\left(\xi_{j}, \eta_{j}, \zeta_{j}\right)=\delta_{i j}, \quad i>m, \\
& 1=\sum_{i>m} N_{i}^{*} .
\end{aligned}
$$


Notice that the $N_{i}^{*}$ functions of (29) satisfy all the conditions needed for the $M_{i}^{*}$ functions of (20).

On the singularity mapping that we are concerned with we further impose the beautiful correspondency to the conventional isoparametric mapping without any contradiction to the preceding arguments. Namely at the limit of $\lambda=1$, the mapping shape functions should be identical to the field shape functions as in (8). and consequently the singularities should be extinguished. We thus naturally put

$$
M_{i}^{*}=N_{i}^{*}, \quad i>m,
$$

which yields

$$
M_{i}=\rho^{1 \lambda} N_{i}, \quad i>m .
$$

We next consider other shape functions contributing to the central edge. Since no singularities are needed in the $z$-directional derivative of our trial function, we have isoparametrically

$$
M_{i}(-1,-1, \zeta)=N_{i}(-1,-1, \zeta), \quad i=1, \ldots, m .
$$

Then (25) can be written as

$$
M_{i}=(1-\rho) N_{i}(-1,-1, \zeta), \quad i=1, \ldots, m .
$$

Our singularity mapping shape functions can thus uniquely be determined by (33) and (35) from the polynomial field shape functions. This is the semi-radial singularity mapping for line singularities. If we eliminate $\rho$ in (33) and (35) by using (19) and (28), then the semi-radial singularity mapping shape functions can directly be connected with the field shape functions such that

$$
\begin{aligned}
M_{i} & =\left\{1-\left(1-\sum_{j=1}^{m} N_{j}\right)^{1 / \lambda}\right\} N_{i}(-1,-1, \zeta), \quad i=1, \ldots, m, \\
M_{i} & =\left(1-\sum_{j=1}^{m} N_{j}\right)^{-1+1 / \lambda} N_{i}, \quad i>m .
\end{aligned}
$$

\subsection{Semi-radial singularity mapping for an 8-node brick}

Consider, for instance, an original 8-node brick element of Fig. 4 with the nodal placement of

$$
\begin{aligned}
& x_{1}=x_{2}=x_{5}=x_{8}=y_{1}=y_{2}=y_{3}=y_{6}=z_{2}=z_{3}=z_{4}=z_{5}=0, \\
& x_{3}=x_{4}=x_{6}=x_{7}=X, \quad y_{4}=y_{5}=y_{7}=y_{8}=Y, \quad z_{1}=z_{6}=z_{7}=z_{8}-Z .
\end{aligned}
$$

We adopt the well-known trilinear interpolation basis by 


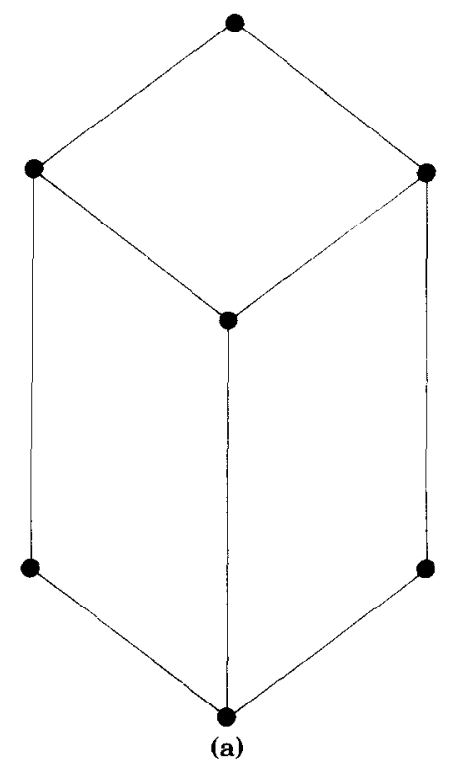

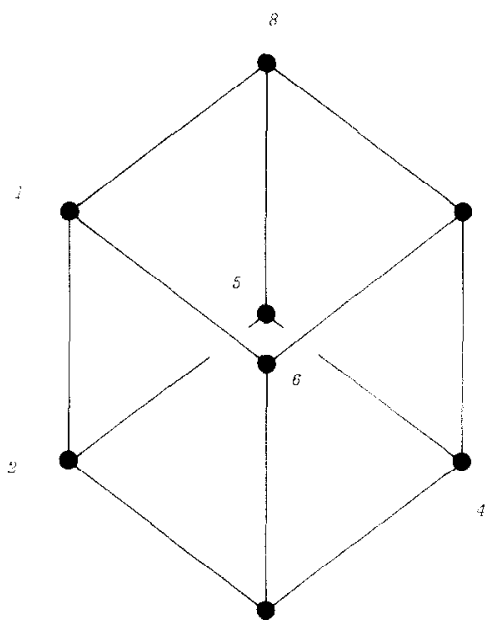

(b)

Fig. 4. The original 8-node brick element geometry. (a) Rectangular brick in the global Cartesian system $(x, y, z)$. (b) Normalized cube in the local parametric system $(\xi, \eta, \zeta)$.

$$
\begin{array}{ll}
N_{1}=\frac{1}{8}(1-\xi)(1-\eta)(1+\zeta), & N_{2}=\frac{1}{8}(1-\xi)(1-\eta)(1-\zeta), \\
N_{3}=\frac{1}{8}(1+\xi)(1-\eta)(1-\zeta), & N_{4}=\frac{1}{8}(1+\xi)(1+\eta)(1-\zeta), \\
N_{5}=\frac{1}{8}(1-\xi)(1+\eta)(1-\zeta), & N_{6}=\frac{1}{8}(1+\xi)(1-\eta)(1+\zeta), \\
N_{7}=\frac{1}{8}(1+\xi)(1+\eta)(1+\zeta), & N_{8}=\frac{1}{8}(1-\xi)(1+\eta)(1+\zeta),
\end{array}
$$

which guarantees the satisfaction of the constant field condition (27).

Then the nondimensional radius is defined as

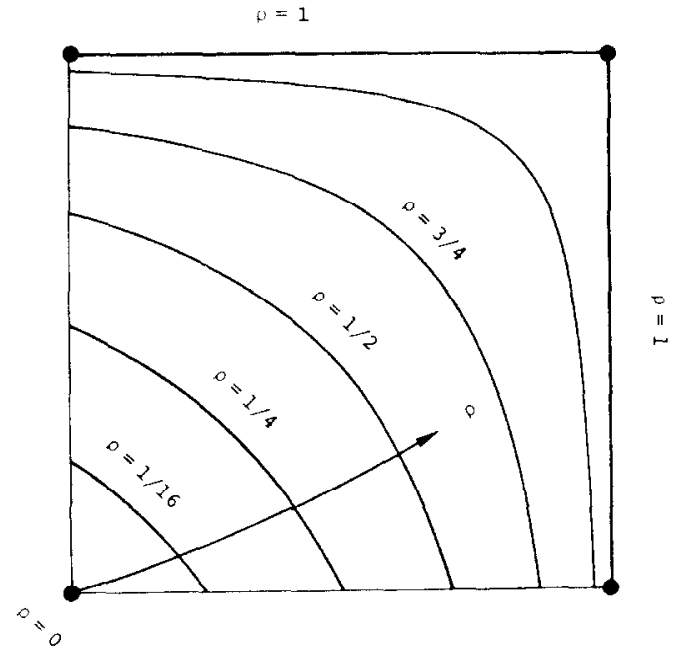

Fig. 5. Typical constant $\rho$ lines on the $\xi$ - $\eta$-plane in linear fracture mechanics due to $\sqrt{\rho}=\frac{1}{4}(3+\xi+\eta-\xi \eta)$. 


$$
\rho^{\wedge}=1-N_{1}-N_{2}=\frac{1}{4}(3+\xi+\eta-\xi \eta) .
$$

Notice that $\rho$ by (40) is independent of $\zeta$ and identical to the nondimensional radius in the quadrilateral semi-radial singularity mapping dealing with the two-dimensional point singularities [21]. Typical constant $\rho$ lines with $\lambda=\frac{1}{2}$ are shown in Fig. 5 on the constant $\zeta$-plane. In particular we emphasize that $\rho$ by (40) is monotone along the ray emanating from the central edge on the constant $\zeta$-plane.

Equations (36) and (37) then yield

$$
\begin{aligned}
& M_{1}=\frac{1}{2}\left[1-\{(3+\xi+\eta-\xi \eta) / 4\}^{1 / \lambda}\right](1+\zeta), \\
& M_{2}=\frac{1}{2}\left[1-\{(3+\xi+\eta-\xi \eta) / 4\}^{1 / \lambda}\right](1-\zeta), \\
& M_{3}=\frac{1}{8}\{(3+\xi+\eta-\xi \eta) / 4\}^{-1+1 / \lambda}(1+\xi)(1-\eta)(1-\zeta), \\
& M_{4}=\frac{1}{8}\{(3+\xi+\eta-\xi \eta) / 4\}^{-1+1 / \lambda}(1+\xi)(1+\eta)(1-\zeta), \\
& M_{5}=\frac{1}{8}\{(3+\xi+\eta-\xi \eta) / 4\}^{-1+1 / \lambda}(1-\xi)(1+\eta)(1-\zeta), \\
& M_{6}=\frac{1}{8}\{(3+\xi+\eta-\xi \eta) / 4\}^{-1+1 / \lambda}(1+\xi)(1-\eta)(1+\zeta), \\
& M_{7}=\frac{1}{8}\{(3+\xi+\eta-\xi \eta) / 4\}^{-1+1 / \lambda}(1+\xi)(1+\eta)(1+\zeta), \\
& M_{8}=\frac{1}{8}\{(3+\xi+\eta-\xi \eta) / 4\}^{-1+1 / \lambda}(1-\xi)(1+\eta)(1+\zeta),
\end{aligned}
$$

Notice that (39)-(41) are adequate for any distorted brick element. The reader can then easily verify that the $N_{i}^{*}$ functions $(i=3, \ldots, 8)$ related to (39) are bounded everywhere within the element. The proportionality of $\rho$ and $r$ is thus realized within the vicinity of the central edge.

For the rectangular brick concerned the semi-radial singularity mapping can simply be written as

$$
\begin{aligned}
& x=\frac{1}{2} X\{(3+\xi+\eta-\xi \eta) / 4\}^{-1+1 / \lambda}(1+\xi), \\
& y=\frac{1}{2} Y\{(3+\xi+\eta-\xi \eta) / 4\}^{-1+1 / \lambda}(1+\eta), \\
& z=\frac{1}{2} Z(1+\zeta) .
\end{aligned}
$$

\subsection{Semi-radial singularity mapping for a 6-node wedge} of

We next consider the original 6-node wedge element of Fig. 6 with the nodal placement

$$
\begin{aligned}
& x_{1}=x_{2}=y_{1}=y_{2}=z_{2}=z_{3}=z_{4}=0, \\
& x_{3}=x_{5}=X_{1}, \quad y_{3}=y_{5}=Y_{1}, \\
& x_{4}=x_{6}=X_{2}, \quad y_{4}=y_{6}=Y_{2}, \quad z_{1}=z_{5}=z_{6}=Z .
\end{aligned}
$$

We adopt the trilinear interpolation basis of (39) but with collapse of nodes 1 and $1_{D}$ and nodes 2 and $2_{D}$ (Fig. 6) such that 

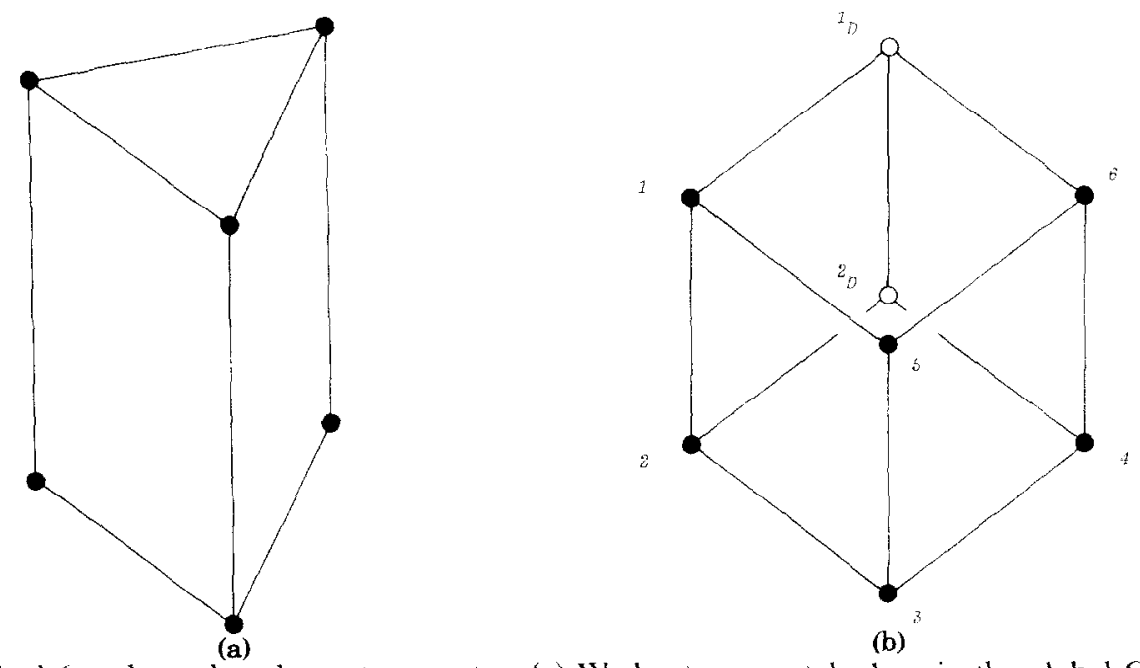

Fig. 6. The original 6-node wedge element geometry. (a) Wedge-type pentahedron in the global Cartesian system $(x, y, z)$. (b) Normalized cube in the local parametric system $(\xi, \eta, \zeta)$ with collapse of nodes 1 and $1_{D}$ and nodes 2 and $2_{D}$.

$$
\begin{array}{ll}
N_{1}=\frac{1}{4}(1-\xi)(1+\zeta), & N_{2}=\frac{1}{4}(1-\xi)(1-\zeta), \\
N_{3}=\frac{1}{8}(1+\xi)(1-\eta)(1-\zeta), & N_{4}=\frac{1}{8}(1+\xi)(1+\eta)(1-\zeta) \\
N_{5}=\frac{1}{8}(1+\xi)(1-\eta)(1+\zeta), & N_{6}=\frac{1}{8}(1+\xi)(1+\eta)(1+\zeta)
\end{array}
$$

The nondimensional radius is then defined as

$$
\rho^{\lambda}=1-N_{1}-N_{2}=\frac{1}{2}(1+\xi)
$$

We remark that $\rho$ by (45) is independent not only of $\zeta$ but also of $\eta$, and the same nondimensional radius appears in the triangular semi-radial singularity mapping dealing with two-dimensional point singularities $[12,20,21]$. Typical constant $\rho$ lines with $\lambda=\frac{1}{2}$ are contoured in Fig. 7 on the constant $\zeta$-plane. Obviously $\rho$ by (45) is monotone along the ray emanating from the central edge.

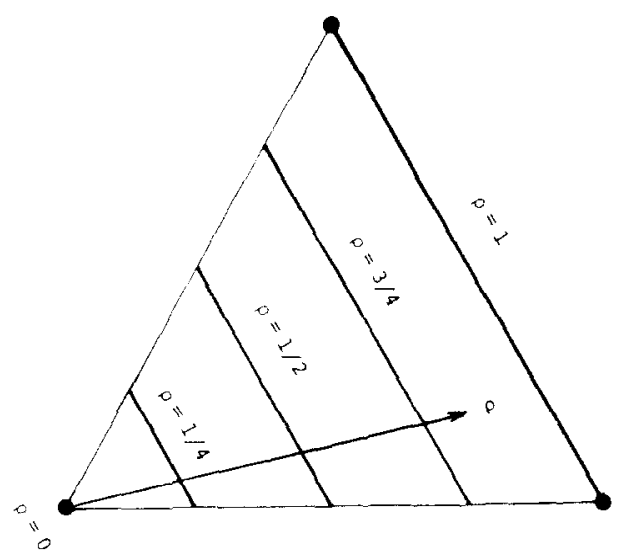

Fig. 7. Typical constant $\rho$ lines on the $\xi$ - $n$-plane in linear fracture mechanics due to $\sqrt{\rho}=\frac{1}{2}(1+\xi)$. 
From (36) and (37) we have

$$
\begin{array}{ll}
M_{1}=\frac{1}{2}\left[1-\{(1+\xi) / 2\}^{1 / \lambda}\right](1+\zeta), & M_{2}=\frac{1}{2}\left[1-\{(1+\xi) / 2\}^{1 / \lambda}\right](1-\zeta), \\
M_{3}=\frac{1}{4}\{(1+\xi) / 2\}^{1 / \lambda}(1-\eta)(1-\zeta), & M_{4}=\frac{1}{4}\{(1+\xi) / 2\}^{1 / \lambda}(1+\eta)(1-\zeta), \\
M_{5}=\frac{1}{4}\{(1+\xi) / 2\}^{1 / \lambda}(1-\eta)(1+\zeta), & M_{6}=\frac{1}{4}\{(1+\xi) / 2\}^{1 / \lambda}(1+\eta)(1+\zeta) .
\end{array}
$$

Equations (44)-(46) are adequate for any distorted rectangular prism. Under the nodal placement of (43) the semi-radial singularity mapping can thus simply be written as

$$
\begin{aligned}
& x-\frac{1}{2}\{(1+\xi) / 2\}^{1 / \lambda}\left\{X_{1}(1-\eta)+X_{2}(1+\eta)\right\}, \\
& y=\frac{1}{2}\{(1+\xi) / 2\}^{1 / \lambda}\left\{Y_{1}(1-\eta)+Y_{2}(1+\eta)\right\}, \\
& z=\frac{1}{2} Z(1+\zeta) .
\end{aligned}
$$

REMARK 4.1 (on the general polynomial interpolations). Again we emphasize that our trial functions are based on the conventional polynomial interpolations in $(\xi, \eta, \zeta)$. Then corresponding to (24) the field shape functions contributing to the central edge satisfy

$$
N_{i}=N_{i}(-1,-1, \zeta) \sum_{j=1}^{m} N_{j}, \quad i=1, \ldots, m
$$

Equations (35) and (36) can thus generally be rewritten as

$$
\begin{aligned}
& M_{i}=(1-\rho) N_{i} /\left(1-\rho^{\lambda}\right), \quad i=1, \ldots, m, \\
& M_{i}=\left\{1-\left(1-\sum_{j=1}^{m} N_{j}\right)^{1 / \lambda}\right\} N_{i} / \sum_{j=1}^{m} N_{j}, \quad i=1, \ldots, m .
\end{aligned}
$$

In the more complicated finite elements of Fig. 1 we always encounter the nondimensional radii by (40) and (45) corresponding to the normalized cube without collapse and with collapse, respectively. Hence $\rho$ by (40) is termed the intrinsic hypercube nondimensional radius, while $\rho$ by (45) is called the intrinsic simplex nondimensional radius. In the semi-radial singularity mapping these intrinsic nondimensional radii should always be used.

\section{Jacobian transformation formulae}

In the general semi-radial singularity mapping the parametric representation can be expressed as

$$
x-x_{0}=\sum_{i>m}\left(x_{i}-x_{0}\right) M_{i} .
$$

Then using (10), (29) and (33) differentiation of (51) gives 


$$
\begin{aligned}
& \partial \boldsymbol{x} / \partial \xi=\sum_{i>m}\left(\boldsymbol{x}_{i}-\boldsymbol{x}_{0}\right)\left\{\rho^{1-\lambda} \partial N_{i} / \partial \xi+(1-\lambda) N_{i}^{*} \partial \rho / \partial \xi\right\} \\
& \partial \boldsymbol{x} / \partial \eta=\sum_{i>m}\left(\boldsymbol{x}_{i}-\boldsymbol{x}_{0}\right)\left\{\rho^{1-\lambda} \partial N_{i} / \partial \eta+(1-\lambda) N_{i}^{*} \partial \rho / \partial \eta\right\} \\
& \partial \boldsymbol{x} / \partial \zeta-(1-\rho) \partial \boldsymbol{x}_{0} / \partial \zeta+\sum_{i>m}\left(\boldsymbol{x}_{i}-\boldsymbol{x}_{0}\right) \rho^{1-\lambda} \partial N_{i} / \partial \zeta
\end{aligned}
$$

Here we assume that $\rho$ is independent of $\zeta$.

\subsection{8-node brick singularity element}

The Jacobian relations inherent to the 8-node brick element of Fig. 4 can explicitly be written as

$$
|\boldsymbol{J}|=\frac{1}{2} \rho^{2-2 \lambda} f_{l} \boldsymbol{Z}
$$

and

$$
\begin{aligned}
& \partial \xi / \partial x=\rho^{\lambda-1}\left\{4 \lambda+(1-\lambda) \rho^{-\lambda}(1-\xi)(1 \mid \eta)\right\} Y / 8 \lambda f_{J}, \\
& \partial \xi / \partial y=-\rho^{\lambda \cdots 1}(1-\lambda) \rho^{-\lambda}\left(1-\xi^{2}\right) X / 8 \lambda f_{J}, \\
& \partial \eta / \partial x=-\rho^{\lambda-1}(1-\lambda) \rho^{-\lambda}\left(1-\eta^{2}\right) Y / 8 \lambda f_{J}, \\
& \partial \eta / \partial y=\rho^{\lambda} \quad\left\{4 \lambda+(1-\lambda) \rho^{\lambda}(1+\xi)(1-\eta)\right\} X / 8 \lambda f_{J}, \\
& \partial \zeta / \partial z=2 / Z \\
& \partial \xi / \partial z=\partial \eta / \partial z=\partial \zeta / \partial x=\partial \zeta / \partial y=0 .
\end{aligned}
$$

Here $|\boldsymbol{J}|$ denotes the Jacobian and the denominator function $f_{J}$ is given by

$$
\begin{aligned}
f_{J} & =\left\{2 \lambda+(1-\lambda) \rho^{-\lambda}(1+\xi \eta)\right\} X Y / 8 \lambda \\
& =\frac{X Y}{4 \lambda} \cdot \frac{2(1+\xi)+2(1+\eta)-(2-\lambda)(1+\xi)(1+\eta)}{2(1+\xi)+2(1+\eta)-(1+\xi)(1+\eta)} .
\end{aligned}
$$

We note that $f_{J}$ by (55) is positive throughout the region of interest [21].

5.2. 6-node wedge singularity element

The transformation formulac associated with the 6-node element of Fig. 6 can be expressed as

$$
|\boldsymbol{J}|=\rho^{2-\lambda} Z T / 8 \lambda
$$

and

$$
\begin{aligned}
& \partial \xi / \partial x=2 \lambda\left(Y_{2}-Y_{1}\right) \rho^{\lambda-1} / T, \quad \partial \xi / \partial y=-2 \lambda\left(X_{2}-X_{1}\right) \rho^{\lambda-1} / T, \\
& \partial \eta / \partial x=-\left\{Y_{1}(1-\eta)+Y_{2}(1+\eta)\right\} \rho^{-1} / T, \\
& \partial \eta / \partial y=\left\{X_{1}(1-\eta)+X_{2}(1+\eta)\right\} \rho^{-1} / T, \\
& \partial \zeta / \partial z=2 / Z, \quad \partial \xi / \partial z=\partial \eta / \partial z=\partial \zeta / \partial x=\partial \zeta / \partial y=0 .
\end{aligned}
$$


Here $T$ denotes twice the area of the triangular surface in the global Cartesian system by

$$
T=X_{1} Y_{2}+X_{2} Y_{1}
$$

\section{Singularity evaluation}

We have defined the semi-radial singularity mapping so that the lowest terms associated with the radius $r$ in our trial function are $\mathrm{O}\left(r^{\lambda}\right)$ within the vicinity of the central edge (except for the $r^{0}$ terms). Therefore, $\mathrm{O}\left(r^{\lambda-1}\right)$ singularities may appropriately appear in $\partial \phi / \partial x$ and $\partial \phi / \partial y$. In this section we present a much more direct proof for the preceding two examples. Hereafter we denote $x$ and $y$ by $x$ since $\partial \phi / \partial z$ has no singularity.

\subsection{8-node brick element}

We consider first the 8-node brick element of Fig. 4. It is clear in (54) that the $\partial \xi / \partial x$ and $\partial \eta / \partial x$ terms have $\mathrm{O}\left(\rho^{\lambda-1}\right)$ singularities. In the partial differential formula of the form

$$
\partial \phi / \partial \boldsymbol{x}=(\partial \phi / \partial \xi)(\partial \xi / \partial \boldsymbol{x})+(\partial \phi / \partial \eta)(\partial \eta / \partial \boldsymbol{x})
$$

the $\partial \phi / \partial \xi$ and $\partial \phi / \partial \eta$ terms are polynomials in $(\xi, \eta, \zeta)$. Hence the first derivatives $\partial \phi / \partial x$ and $\partial \phi / \partial y$ have $\mathrm{O}\left(\rho^{\lambda-1}\right)$, i.e., $\mathrm{O}\left(r^{\lambda-1}\right)$ singularities as expected.

The strain energy within the element can be estimated by

$$
E=\int_{-1}^{1} \int_{-1}^{1} \int_{-1}^{1}(\partial \phi / \partial \boldsymbol{x})^{2}|\boldsymbol{J}| \mathrm{d} \xi \mathrm{d} \eta \mathrm{d} \zeta
$$

The $\partial \phi / \partial x$ term contains $O\left(\rho^{\lambda-1}\right)$ singularities, but fortunately we have $O\left(\rho^{2-2 \lambda}\right)$ in the Jacobian of (53). Thus the integrand of (60) has no singularity, which ensures the boundedness of the strain energy.

\subsection{6-node wedge element}

In the 6-node wedge element of Fig. 6(57) describes that $\partial \xi / \partial x$ is a product of the $\rho^{\lambda-1}$ term and another polynomial while $\partial \eta / \partial x$ has the $\rho^{-1}$ term. However, $\partial \phi / \partial \eta$ takes a value of strictly zero at $\xi=-1$, and hence it has a factor of $(1+\xi)$, i.e. $\rho^{\lambda}$. It is now clear in (59) that the first derivatives $\partial \phi / \partial x$ and $\partial \phi / \partial y$ have the $\rho^{\lambda-1}$ factor.

Equation (56) further ensures that the integrand of $(60)$ is a product of the $(1+\xi)$ term and another polynomial, and consequently the strain energy is bounded.

\section{Element familization}

Once the appropriate mapping shape functions are determined for the original nodal placement it is easy to place other additional nodes without changing the element geometry and also without influence upon the developed transformation formulae. Our semi-radial singularity mapping element can thus systematically be familized with the known polynomial 
interpolation bases. Incorporation of the semi-radial singularity mapping family into the existing finite element programs can then easily be attained only by the minor modification of the Jacobian transformation routine.

We present here a few elaborate elements having the reproducibility of $1, \rho^{\lambda}, \rho^{2 \lambda}, \rho^{\lambda-1} r$, $\rho^{2 \lambda-\gamma} \boldsymbol{r}^{\prime}, z$ and $z^{2}$ with the necessary minimum nodal placement. In linear fracture mechanics of $\lambda=\frac{1}{2}$ the reproducibility of these terms ensures the satisfaction of the global linear field reproducibility of (9). It is further associated with the polynomial completeness of degree (wo in $(x, y, z)$ in the isoparametric mapping at the limit of $\lambda=1$.

\subsection{The brick singularity family with the rectangular interpolations}

Consider, for example, the 22-node brick element of Fig. 8 . Then the field shape function are given by

$$
\begin{aligned}
& N_{0,0,0}=-\frac{1}{8}(1-\xi)(1-\eta)(1-\zeta)(1+\zeta-\xi \eta), \\
& N_{0.0 .1}=\frac{1}{4}(1-\xi)(1-\eta)\left(1-\zeta^{2}\right) \text {, } \\
& N_{0,0.2}--\frac{1}{8}(1-\xi)(1-\eta)(1+\zeta)(1-\zeta-\xi \eta) \text {, } \\
& N_{0,1,0}=-\frac{1}{4} \xi(1-\xi)\left(1-\eta^{2}\right)(1-\zeta) \text {. } \\
& N_{0,1.2}=-\frac{1}{4} \xi(1-\xi)\left(1-\eta^{2}\right)(1+\zeta) \text {, } \\
& N_{0,2,0}=-\frac{1}{8}(1-\xi)(1+\eta)(1-\zeta)(1+\zeta+\xi \eta) \text {. } \\
& N_{0,2,1}=\frac{1}{4}(1-\xi)(1+\eta)\left(1-\zeta^{2}\right) \text {, } \\
& N_{0,2,2}=-\frac{1}{8}(1-\xi)(1+\eta)(1+\zeta)(1-\zeta+\xi \eta) \text {, } \\
& N_{1,0.0}=-\frac{1}{4}\left(1-\xi^{2}\right) \eta(1-\eta)(\mathrm{I}-\zeta) \text {. } \\
& N_{1,0,2}--\frac{1}{4}\left(1-\xi^{2}\right) \eta(1-\eta)(1+\zeta) \text {, } \\
& N_{1,1,0}=\frac{1}{2}\left(1-\xi^{2}\right)\left(1-\eta^{2}\right)(1-\zeta) \text {. } \\
& N_{1,1,2}=\frac{1}{2}\left(1-\xi^{2}\right)\left(1-\eta^{2}\right)(1+\zeta) \text {. } \\
& N_{1,2,0}=\frac{1}{4}\left(1-\xi^{2}\right) \eta(1+\eta)(1-\zeta) \text {, } \\
& N_{1.2 .2}=\frac{1}{4}\left(1-\xi^{2}\right) \eta(1+\eta)(1+\zeta) \text {, } \\
& N_{2,0,0}=-\frac{1}{8}(1+\xi)(1-\eta)(1-\zeta)(1+\zeta+\xi \eta) \text {, } \\
& N_{2,0,1}=\frac{1}{4}(1+\xi)(1-\eta)\left(1-\zeta^{2}\right) \text {, } \\
& N_{2,0,2}=-\frac{1}{8}(1+\xi)(1-\eta)(1+\zeta)(1-\zeta+\xi \eta) \text {. } \\
& N_{2,1,0}=\frac{1}{4} \xi(1+\xi)\left(1-\eta^{2}\right)(1-\zeta) \text {. } \\
& N_{2,1,2}=\frac{1}{4} \xi(1+\xi)\left(1-\eta^{2}\right)(1+\zeta) \text {, } \\
& N_{2,2,0}=-\frac{1}{8}(1+\xi)(1+\eta)(1-\zeta)(1+\zeta-\xi \eta) \text {, } \\
& N_{2,2,1}=\frac{1}{4}(1+\xi)(1+\eta)\left(1-\zeta^{2}\right) \text {, } \\
& N_{2,2,2}=-\frac{1}{8}(1+\xi)(1+\eta)(1+\zeta)(1-\zeta-\xi \eta) .
\end{aligned}
$$




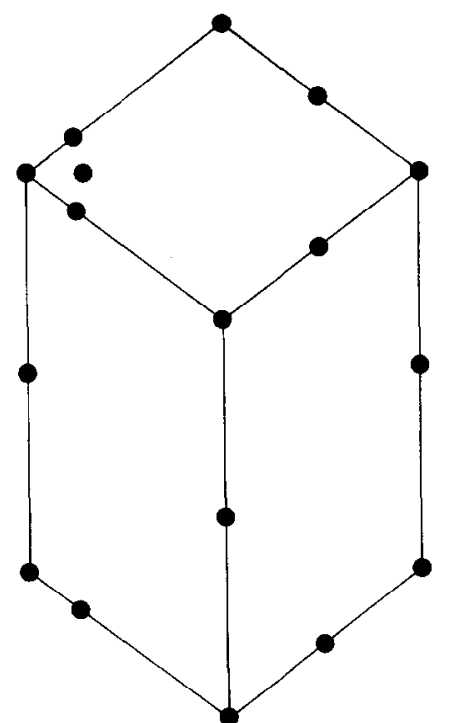

(a)

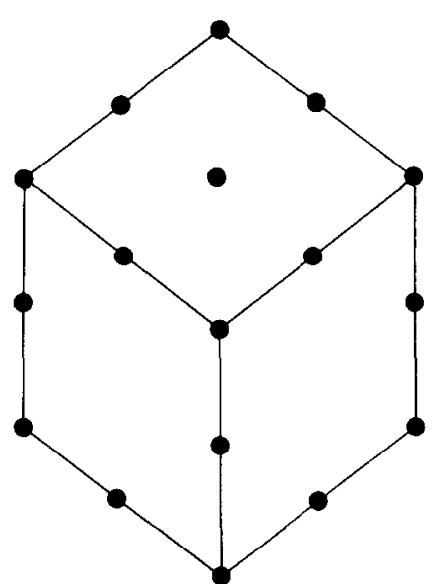

(b)

Fig. 8. The 22-node brick element geometry. (a) Rectangular prism in the global Cartesian system $(x, y, z)$. (b) Normalized cube in the local parametric system $(\xi, \eta, \zeta)$. Five face and internal nodes on $\zeta=0$ are eliminated from the well-known 27-node rectangular Lagrange element [2].

Here the triple index $(i, j, k)$ designates a node having the parametric coordinates of $(i-1, j-$ $1, k-1)$. This elaborate trial function space contains the original space by (39), and hence the reproducibility of $1, \rho^{\lambda}, \rho^{\lambda-1} r$ and $z$ inherent to the original trial function is automatically preserved. For the systematical development of (61), see [6].

Notice that (61) satisfies the $\rho^{2 \lambda}$ reproducibility such that

$$
\begin{aligned}
(3+\xi+\eta-\xi \eta)^{2} / 16= & 1-\left(N_{0,0,0}+N_{0,0,1}+N_{0,0,2}\right)-\frac{3}{4}\left(N_{0,1,0}+N_{0,1,2}+N_{1,0,0}+N_{1,0,2}\right) \\
& -\frac{7}{16}\left(N_{1,1,0}+N_{1,1,2}\right) .
\end{aligned}
$$

In the same manner we can prove that $\rho^{2 \lambda-2} r^{2}$ and $z^{2}$ are reproducible in (61) under the parametric representation (42).

Thus the general rectangular polynomial interpolations yield a series of brick-type singularity elements. In particular we note that not the Serendipity but the regular Lagrange nodal placement is preferable on the surface of $\zeta= \pm 1$.

\subsection{The wedge singularity family with the collapsed rectangular interpolations}

If we degenerate the surface of $\xi=-1$ into the central edge of $\xi=\eta=-1$, then the conventional rectangular interpolations can also be applied to the more elaborate wedge elements. In the preceding example such degenerating techniques give

$$
\begin{aligned}
& N_{0, *, 0}=N_{0,0,0}+N_{0,1,0}+N_{0,2,0}=-\frac{1}{4}(1-\xi)(1-\zeta)(1+\xi+\zeta), \\
& N_{0, *, 1}=N_{0,0,1}+N_{0,2,1}=\frac{1}{2}(1-\xi)\left(1-\zeta^{2}\right), \\
& N_{0, *, 2}=N_{0,0,2}+N_{0,1,2}+N_{0,2,2}=-\frac{1}{4}(1-\xi)(1+\zeta)(1+\xi-\zeta) .
\end{aligned}
$$

The other field shape functions in (61) are valid in this case also. 


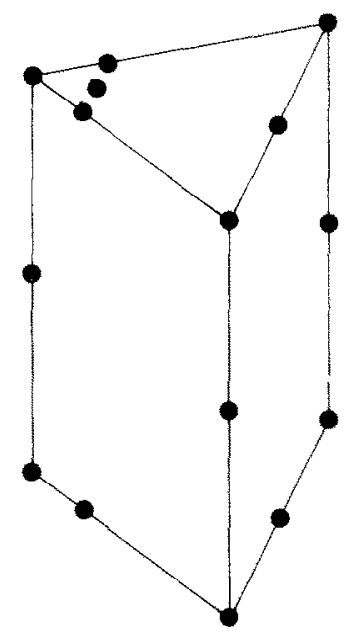

Fig. 9. The 17-node wedge element in the global Cartesian system $(x, y, z)$. In the 22-node cube of Fig. $8(\mathrm{~b})$ the $\xi=-1$ plane in degenerated into the central edge of $\xi=\eta=-1$.

Thus we have the 17-node wedge element of Fig. 9, the trial function space of which involves $1, \rho^{\lambda}, \rho^{2 \lambda}, \rho^{\lambda-1} r, \rho^{2 \lambda-2} r^{2}, z$ and $z^{2}$ under the semi-radial singularity mapping of (47). We remark that the regular Lagrange nodal placement is desirable on the surface of $\zeta= \pm 1$ instead of the Serendipity one.

\subsection{The wedge singularity family with the triangular interpolations}

Let us introduce another local parametric system $\left(\omega_{1}, \omega_{2}, \omega_{3}, \zeta\right)$ defined by

$$
\omega_{1}=\frac{1}{2}(1-\xi), \quad \omega_{2}=\frac{1}{4}(1+\xi)(1-\eta), \quad \omega_{3}=\frac{1}{4}(1+\xi)(1+\eta) .
$$

Here $\omega_{1}, \omega_{2}$ and $\omega_{3}$ are the 2-D volume (i.e. area) coordinates related to $\xi$ and $\eta$ [12].

For a 15-node wedge element of Fig. 10 where two face nodes are eliminated in the previous 17-node wedge, we apply the famous triangular interpolations by using $\omega_{1}, \omega_{2}$ and $\omega_{3}$. Then the field shape functions can be written as

$$
\begin{aligned}
& N_{0, *, 0}=-\frac{1}{2} \omega_{1}(1-\zeta)\left(2-2 \omega_{1}+\zeta\right), \quad N_{0, *, 1}=\omega_{1}\left(1-\zeta^{2}\right), \\
& N_{0,+, 2}=-\frac{1}{2} \omega_{1}(1+\zeta)\left(2-2 \omega_{1}-\zeta\right), \quad N_{1,0,0,0}=2 \omega_{1} \omega_{2}(1-\zeta), \\
& N_{1,0,2}=2 \omega_{1} \omega_{2}(1+\zeta), \quad N_{1,2,0)}=2 \omega_{3} \omega_{1}(1-\zeta), \quad N_{1,2,2}=2 \omega_{3} \omega_{1}(1+\zeta), \\
& N_{2,0,0}=-\frac{1}{2} \omega_{2}(1-\zeta)\left(2-2 \omega_{2}+\zeta\right), \quad N_{2,0,1}=\omega_{2}\left(1-\zeta^{2}\right), \\
& N_{2,0,2}=-\frac{1}{2} \omega_{2}(1+\zeta)\left(2-2 \omega_{2}-\zeta\right), \quad N_{2,1,0}=2 \omega_{2} \omega_{3}(1-\zeta), \\
& N_{2,1,2}=2 \omega_{2} \omega_{3}(1+\zeta), \quad N_{2,2,0}=-\frac{1}{2} \omega_{3}(1-\zeta)\left(2-2 \omega_{3}+\zeta\right), \\
& N_{2,2,1,1}=\omega_{3}\left(1 \quad \zeta^{2}\right), \quad N_{2,2,2}=-\frac{1}{2} \omega_{3}(1+\zeta)\left(2-2 \omega_{3}-\zeta\right) .
\end{aligned}
$$




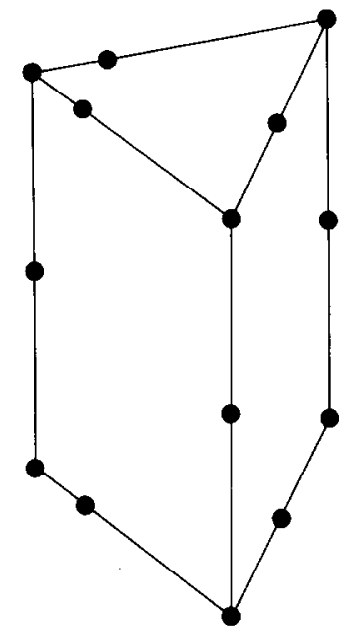

(a)

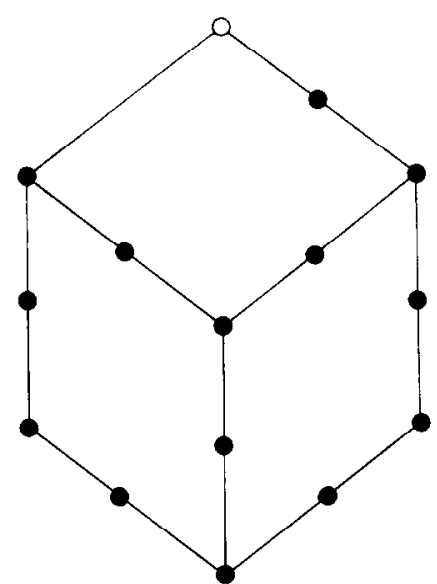

(b)

Fig. 10. The 15-node wedge element geometry. (a) Triangular prism in the global Cartesian system $(x, y, z)$. (b) Normalized cube composed of only side and corner nodes in the local parametric system $(\xi, \eta, \zeta)$ with collapse.

$$
\begin{aligned}
& N_{0, *, 0}=-\frac{1}{4}(1-\xi)(1-\zeta)(1+\xi+\zeta), \quad N_{0, *, 1}=\frac{1}{2}(1-\xi)\left(1-\zeta^{2}\right), \\
& N_{0, *, 2}=-\frac{1}{4}(1-\xi)(1+\zeta)(1+\xi-\zeta), \quad N_{1,0,0}=\frac{1}{4}\left(1-\xi^{2}\right)(1-\eta)(1-\zeta), \\
& N_{1,0,2}=\frac{1}{4}\left(1-\xi^{2}\right)(1-\eta)(1+\zeta), \quad N_{1,2,0}=\frac{1}{4}\left(1-\xi^{2}\right)(1+\eta)(1-\zeta), \\
& N_{1,2,2}=\frac{1}{4}\left(1-\xi^{2}\right)(1+\eta)(1+\zeta), \\
& N_{2,0,0}=-\frac{1}{16}(1+\xi)(1-\eta)(1-\zeta)(3-\xi+\eta+2 \zeta+\xi \eta), \\
& N_{2,0,1}=\frac{1}{4}(1+\xi)(1-\eta)\left(1 \quad \zeta^{2}\right), \\
& N_{2,0,2}=-\frac{1}{16}(1+\xi)(1-\eta)(1+\zeta)(3-\xi+\eta-2 \zeta+\xi \eta), \\
& N_{2,1,0}=\frac{1}{8}(1+\xi)^{2}\left(1-\eta^{2}\right)(1-\zeta), \quad N_{2,1,2}=\frac{1}{8}(1+\xi)^{2}\left(1-\eta^{2}\right)(1+\zeta), \\
& N_{2,2,0}=-\frac{1}{16}(1+\xi)(1+\eta)(1-\zeta)(3-\xi-\eta+2 \zeta-\xi \eta), \quad N_{2,2,1}=\frac{1}{4}(1+\xi)(1+\eta)\left(1-\zeta^{2}\right) \\
& N_{2,2,2,}=-\frac{1}{16}(1+\xi)(1+\eta)(1+\zeta)(3-\xi-\eta-2 \zeta-\xi \eta) .
\end{aligned}
$$

It is then easy to verify that the constant, $\rho^{\lambda}, \rho^{2 \lambda}, \rho^{\lambda-1} r, \rho^{2 \lambda-2} \boldsymbol{r}^{2}, z$ and $z^{2}$ terms are reproducible in our trial function based on the interpolation basis by (66) under the semi-radial singularity mapping of (47). In this example the $\rho^{2 \lambda}$ reproducibility can be expressed as

$$
\begin{aligned}
\frac{1}{4}(1+\xi)^{2}= & N_{2,0,0}+N_{2,0,1}+N_{2,0,2}+N_{2,1,0}+N_{2,1,2}+N_{2,2,0} \\
& +N_{2,2,1}+N_{2,2,2}+\frac{1}{4}\left(N_{1,0,0}+N_{1,0,2}+N_{1,2,0}+N_{1,2,2}\right)
\end{aligned}
$$

We note that the preceding collapsed rectangular interpolations in $(\xi, \eta)$ require 17 nodes to realize the concerning reproducibility, while 15 nodes are enough according to the triangular interpolations in $\left(\omega_{1}, \omega_{2}, \omega_{3}\right)$. Thus applications of the famous complete Lagrange interpolations for the simplex $[9,10]$ may in general be preferable to the wedge-type finite elements. 


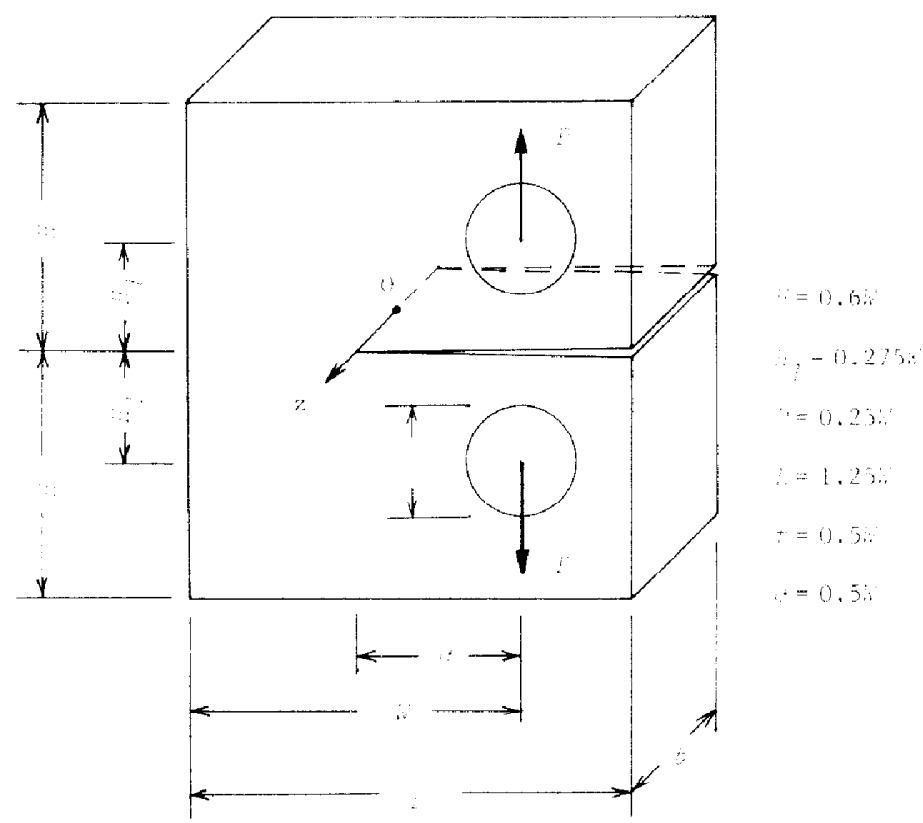

(a)

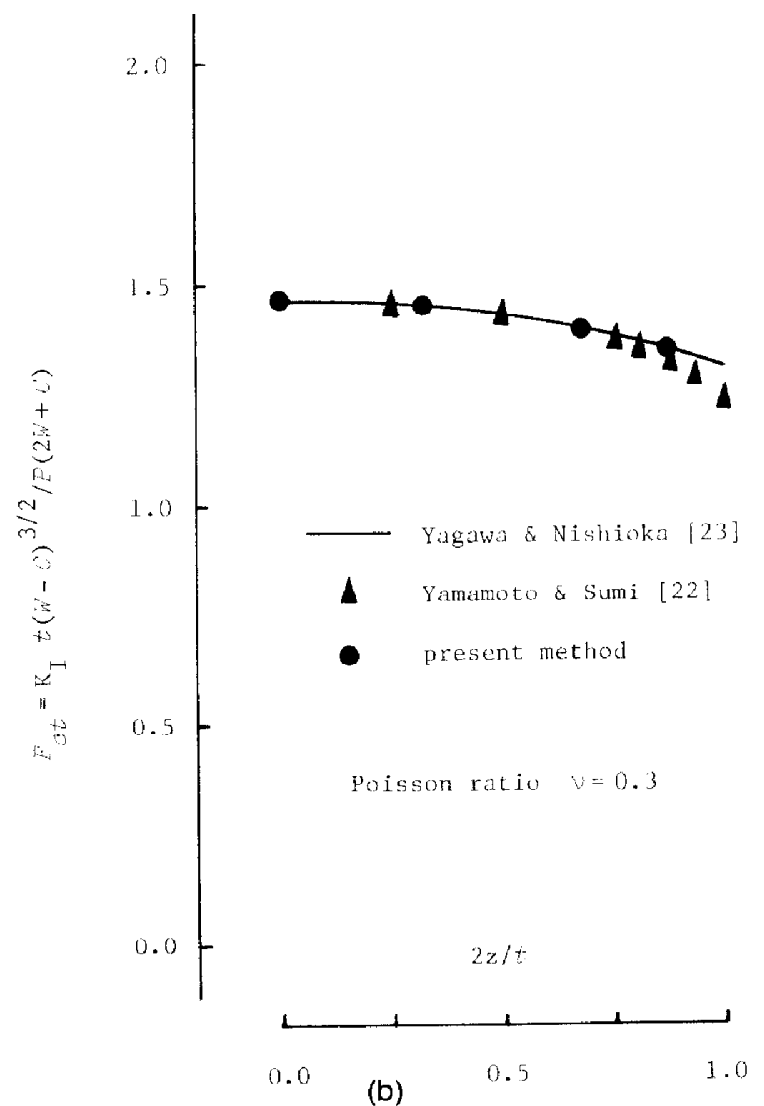

Fig. 11. Compact tension specimen. (a) Specimen geometry. (b) Stress intensity factor across the thickness. 


\section{Numerical example and concluding remarks}

The problem we have solved for verifying our mapping is the compact tension specimen of Fig. 11(a). Computed stress intensity factors are depicted in Fig. 11(b) where wedge elements of Fig. 9 are used. The results are in good agreement with those by Yamamoto et al. [22] and Yagawa et al. [23] due to sophisticated superposition methods. We emphasize that the semi-radial singularity mapping for line singularities can easily be incorporated into any existing finite-element program with only minor modification of the Jacobian transformation routine.

In the singularity elementology, introduction of the nondimensional radius $\rho$ on behalf of the Euclidean radius $r$ is of great significance. Naturally $\rho$ must resemble $r$ as close as possible, and hence the intrinsic hypercube nondimensional radius or the intrinsic simplex one should always be used. It is demonstrated that the $\rho^{\lambda}$ reproducibility plays an important role in the semi-radial singularity mapping. We further pay special attention to the reproducibility of the $\rho^{\lambda}$ and $\rho^{\lambda-1} \boldsymbol{r}$ expansion terms in selecting more elaborate trial function spaces. Therefore, applications of the regular Lagrange family for the cube developed by Argyris $[1,2]$ as well as the complete Lagrange family for the simplex also clarified by Argyris $[7,9]$ are of great importance.

In linear fracture mechanics of $\lambda=\frac{1}{2}$ our elaborate elements of Figs. 8-10 realize the reproducibility of $x, y$ and $z$ by (9). It is rather difficult to separate the effects due to the reproducibility of $\rho^{2 \lambda}$ (i.e. $\rho$ ) and $\rho^{2 \lambda-2} r^{2}$ from those due to the global linear field reproducibility related to $x$ and $y$. For general $\lambda$ (yielding noninteger $1 / \lambda$ ) the $x$ and $y$ terms are never reproducible in our semi-radial singularity mapping elements. However, yet we have no consensus on the necessity of the global linear field reproducibility in general fracture mechanics.

Crack elements with line singularities are introduced in [16,24-26] by combining the twodimensional singular space with the perpendicular polynomial space, say in the form of a tensor product. They are applicable only to the constant thickness platc with straight crack tip line. We remark that our semi-radial singularity mapping is designed so that line singularities along the curved crack tip can be treated even for a variable thickness wall. Evidently, our mapping shape functions are not always separated in variables.

In some cases $\lambda$ could also vary across the thickness. It is noted that the semi-radial singularity mapping is adequate even for $\lambda=\lambda(\zeta)$ but with appropriately modified transformation formulae.

\section{References}

[1] J.H. Argyris, K.E. Buck, I. Fried, G. Mareczek and D.W. Scharpf, Some new elements for matrix displacement methods, Proc. 2nd Conf. Matrix Methods in Structural Mechanics, Air Force Inst. Tech., Wright Patterson Air Force Basc, OH, 1968.

[2] J.H. Argyris and I. Fried, The LUMINA element for the matrix displacement method, Aero, J. 72 (1968) 514-517.

[3] R.L. Taylor, On completeness of shape functions for finite element analysis, Internat. J. Numer. Meths. Engrg. 4 (1970) 17-22.

[4] O.C. Zienkiewicz, The Finite Element Method (McGraw-Hill, London, 3rd ed., 1977). 
[5] A.A. Ball, The interpolation function of a general Serendipity rectangular element, Internat. J. Numer. Meths. Engrg. 15 (1980) 773-778.

[6] M. Okabe, Y. Yamada and I. Nishiguchi, Reconsideration of rectangular Lagrange families with hierarchy ranking bases, Comput. Meths. Appl. Mech. Engrg. 23 (1980) 369-390.

[7] J.H. Argyris, Triangular elements with linearly varying strain for the matrix displacement method, J. Roy. Aero. Soc. 69 (1965) 711-713.

[8] B.F. de Veubeke, Displacement and equilibrium models in the finite element method, in: O.C. Zienkiewic/. and G.S. Holister, eds., Stress Analysis (Wiley, New York, 1965) Ch. 9.

[9] J.H. Argyris, I. Fried and D.W. Scharpf, The TET20 and TEA 8 elements for the matrix displacement method. Aero. J. 72 (1968) 618-625.

[10] P. Silvester, Higher order polynomial triangular finite elements for potential problems. Internat. J. Numer. Meths. Engrg. 7 (1969) 849-861.

[11] P.G. Ciarlet and P.A. Raviert, General Lagrange and Hermite interpolation in $\mathbb{R}^{\prime \prime}$ with applications to finite element methods, Arch. Rational Mech. Anal. 46 (1972) 177-199.

[12] M. Okabe and N. Kikuchi, Some general Lagrange interpolations over simplex finite elements with reference to derivative singularities, Comput. Meths. Appl. Mech. Engrg. 28 (1981) 1-25.

[13] W.S. Blackburn, Calculation of stress intensity factors, in: J.R. Whiteman, ed.. The Mathematics of Finite Elements and Applications ( $A$ cademic Press, London, 1973) 327-336.

[14] J.E. Akin. The generation of elements with singularities, Internat. J. Numer. Meths. Engrg. 10 (1976) $1249-1259$.

[15] D.M. Tracey and T.S. Cook. Analysis of power type singularities using finite elements. Internat. J. Numer. Meths. Engrg. 11 (1977) 1225-1233.

[16] M. Stern, Families of consistent conforming elements with singular derivative fields. Internat. J. Numer. Meths. Engrg. 14 (1979) 409-421.

[17] P.D. Henshell and K.G. Shaw, Crack tip finite elements are unnecessary, Internat. J. Numer. Meths. Engrg. 9 (1975) 495-507.

[18] R.S. Barsoum, On the use of isoparametric finite elements in linear fracture mechanics, Internat. J. Numer. Meths. Engrg. 10 (1976) 25-37.

[19] A. Peano, A. Pasini, R. Riccioni and L. Sardella, Adaptive approximations in finite element structural analysis, Computers and Structures 10 (1979) 333-342.

[20] Y. Yamada, Y. Ezawa, I. Nishiguchi and M. Okabe, Reconsiderations on singularity or crack tip elements, Internat. J. Numer. Meths. Engrg. 14 (1979) 1525-1544.

[21] M. Okabe, Fundamental theory of the semi-radial singularity mapping with applications to fracture mechanics, Comput. Meths. Appl. Mech. Engrg. 26 (1981) 53-73.

[22] Y. Yamamoto and Y. Sumi, Stress intensity factor for three-dimensional cracks, Proc. 14th Internat. Congress Theor. Appl. Mech., Delft, 1976.

[23] G. Yagawa and T. Nishioka, Three-dimensional finite element analysis for through-wall crack in thick plate, Internat. J. Numcr. Meths. Engrg. 12 (1978) 1295-1310.

[24] J.E. Akin, Elements for problems with line singularities, in: J.R. Whiteman, ed., The Mathematics of Finite Elements and Applications, Vol. III (Academic Press, London, 1978) 65-75.

[25] T.J.R. Hughes and J.E. Akin, Techniques for developing 'special' finite element shape functions with particular reference to singularities, Internat. J. Numer. Meths. Engrg. 15 (1980) 733-751.

[26] A.R. Ingraffea and C. Manu, Stress-intensity factor computation in three dimensions with quarter-point elements. Internat. J. Numer. Meths. Engrg. 15 (1980) 1427-1445. 